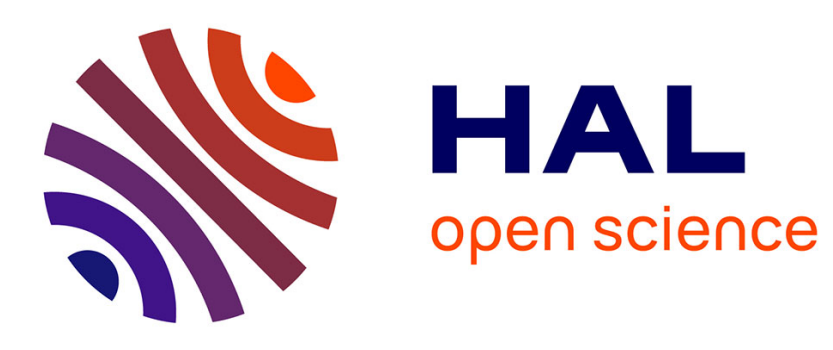

\title{
Ground target finding mechanism for unmanned aerial vehicles to secure crop field data
}

\author{
Mohammad Ammad Uddin, Muhammad Ayaz, Ali Mansour, El-hadi \\ Aggoune, Ahmad Hani El Fawal, Imran Razzak
}

\section{- To cite this version:}

Mohammad Ammad Uddin, Muhammad Ayaz, Ali Mansour, El-hadi Aggoune, Ahmad Hani El Fawal, et al.. Ground target finding mechanism for unmanned aerial vehicles to secure crop field data. Transactions on emerging telecommunications technologies, 2021, 32 (3), pp.e4210. 10.1002/ett.4210 . hal-03126980

\section{HAL Id: hal-03126980 \\ https://hal-ensta-bretagne.archives-ouvertes.fr/hal-03126980}

Submitted on 9 Feb 2021

HAL is a multi-disciplinary open access archive for the deposit and dissemination of scientific research documents, whether they are published or not. The documents may come from teaching and research institutions in France or abroad, or from public or private research centers.
L'archive ouverte pluridisciplinaire HAL, est destinée au dépôt et à la diffusion de documents scientifiques de niveau recherche, publiés ou non, émanant des établissements d'enseignement et de recherche français ou étrangers, des laboratoires publics ou privés. 


\title{
Ground Target Finding Mechanism for Unmanned Aerial Vehicles to Secure Crop Field Data
}

\author{
Mohammad Ammad Uddin', 2, Muhammad Ayaz, 2, Ali Mansour ${ }^{2}$, el-Hadi M. Aggoune1, Ahmad Hani El \\ Fawal $^{2}$, Imran Razzak ${ }^{3}$ \\ ${ }^{1}$ Sensor Networks and Cellular Systems (SNCS) Research Center, University of Tabuk, Tabuk, KSA \\ ${ }^{2}$ Lab STICC, ENSTA Bretagne, Brest, France \\ ${ }^{3}$ Deakin University Australia \\ * Correspondence: mohammad.ammad-uddin@ensta-bretagne.org
}

\begin{abstract}
Unmanned aerial vehicles (UAVs) are being used in many military and civil applications while their use along wireless sensors is emerging abruptly as it upgrades the network in terms of reliability, lifetime, and connectivity. The system performance and throughput can be further enhanced if the UAVs in use are capable to locate ground sensor nodes as a means to address them smartly. In typically localization, the direction of arrival of incoming signals from a target node is measured by using a uniformed linear array (ULA) of antennas having signal processing capabilities. However, adding numerous antennas (transmitter receivers set) on a UAV is an extra burden which ultimately effects its speed, flight time, agility and payload capacity. Considering the issue, a typical ULA antenna system is modeled by using virtual phased array (VPA) antenna. In the proposed VPA, a single antenna installed on a moving UAV will achieve the desire results. The well-known multiple signal classification algorithm is modified to adapt this virtual antenna system. Four extra modules including calibrator, virtual antenna, rectifier and adjustment are added in classical MUSIC algorithm to make it enable to work with single antenna. The proposed system mitigates the existing challenges of physical ULA and improves system throughput. Furthermore, due to its virtualness, VPA can adjust the number of antenna elements and inter-element spacing, which adds multi-frequency support and adaptive precision mechanism. A simulation model is developed in MATLAB to verify the performance of the VPA where the proposed system is evaluated against different scenarios with a varying number of parameters. It is found that VPA gives the same resolution and precision as a physical ULA antenna but it is more flexible, user-friendly, cost-effective, reliable, and most importantly, does not affect the UAV characteristics.
\end{abstract}

Keywords: Unmanned Aerial Vehicle, Target Finding, Localization, Wireless Sensors, Smart Agriculture.

\section{Introduction}

Unmanned aerial vehicles (UAVs) are being used in a variety of civil [1] and military [2] applications nowadays. The use of UAVs as attack/defense line [3], aerial targets for training [4], mining/demining [5], and law enforcement [6] are the examples for military usage, while the use of UAVs in archaeology [7], cargo/passenger transport, maintenance, healthcare [8][9], filmmaking [10], journalism [11], scientific research, search and rescue [12], surveying [13], pollution monitoring [14], oil/gas/mineral exploration [15], disaster relief [16], agriculture [17][18], light show [19], connection of IoTs with cloud [20][21][22], hobby, and recreation are examples of civil applications. In this research, UAVs are used to develop an immediate communication infrastructure in a remote agriculture field to collect data where infrastructure is not available. Furthermore, they resolve many other datagathering problems as well as they are economical in deployment cost, require no preinstalled infrastructure, UAV can visit a sensor node more closely and can approach areas which are difficult or life-threating for humans to get to. An UAV having capability to detect and localize field sensor nodes correctly can perform data collection more efficiently. Crop sensors are mostly not equipped with global positioning system (GPS) modules in an effort to save cost and battery life. Furthermore, in the case of static/non-moving sensor nodes, implanting GPS modules in them is not recommended, as monitoring the same location every time is not practical, especially in the case of energy-sensitive sensor nodes. In such a type of sensors deployment scenario, all the liability shifts to the UAV to search a node, share information and collect data.

In this paper, the use of a UAV is taken as the only mean of communication among large number of heterogeneous sensing nodes scattered in many crop fields. In this scenario, the energy of a UAV is required to 
be preserved, which will allow the UAV to operate for longer periods and cover more area of data collection. To achieve this target, a virtual phased array (VPA) is proposed in which a single antenna is installed over moving UAV to construct a virtual linear array. This VPA will be smaller in size, lighter in weight, and more energyefficient as compared to a physical antenna. Furthermore, multi-frequency support can be achieved by reconfigurable inter-elements spacing, and adaptive steering mechanism becomes possible due to the variability of a number of onboard antenna elements. The other benefits are a lesser effect of mutual coupling and induced current between antenna elements. A number of localization techniques are proposed by available researches. We can categorize these techniques in to two broad groups 1) GPS based and GPS Free 2) centralized and distributed.

GPS-based localization has been discussed in [23][24] while, GPS-free is described in [25] and [26]. Both GPS-enabled and GPS-free schemes have their own pros and cons in terms of energy efficiency, complexity, processing, and weight/size of equipment.

In centralized schemes [27]-[29], a base station determines the distances/locations of all the nodes in its range based on hop count or received signal strength indicator (RSSI) values. This central node periodically sends location information of each node. In distributed scheme [30], [31] position is calculated by sensor nodes individually by interacting directly with anchor nodes or estimated based on distance from reference node or through GPS-sensing. Centralized-based algorithms more economical in terms of computation and energy but issue is bottleneck and connectivity.

The distributed localization schemes can be further divided into two classes. The first one is range-based [32]. In this approach characteristics of information signals, like angle of arrival (AoA), time of arrival (ToA), or time difference of arrival (TDoA), is used to estimate the location of target nodes. While, the other one is range free method [33], location of a target node is estimated by absolute point-to-point distances between them. Several location-aware anchors nodes also called seed nodes are planted in the entire WSN to broadcast periodically location information through control messages. Based on these control messages sensor nodes estimate their own locations by observing the traces that are left on these messages as a result of multiple hops and channel characteristics. It is observed in most of the existing localization techniques that location is calculated or estimated either by Lateration, Angulation, RSSI, ToA, AoA or TDoA.

In lateration method, the location of a target node is calculated based on the knowledge of two or more reference points, positions and distances. In the case of the angulation method, at least two angles of a target node (un-localized) are measured to estimate its position based on the position of the source (localized) nodes. In many research studies RSSI-based localization is discussed, including [34], [35]. It is a self-organized localization scheme that works well only in outdoor environment. The constraint of this scheme is its power utilization because it needs to forward massive information to the central unit. The propagation speed and time of radio signals between the anchor node and the target node are measured to calculate the location of a node. This type of localization is called ToA or ToF (time of flight) based localization [36]. The one-way propagation time of a signal between the transmitter and the receiver is called ToA, which can be multiplied with the propagation speed to get the distance of the target node. As discussed earlier, the synchronization problem that exists in one-way ToA can be avoided in two-way time difference of arrival TDoA [36] [37] in which the distance between the transmitter and the receiver is measured using two signals of different velocities.

When radio wave hits an array of antenna, angle of arrival (AoA) technique can be used to find out the direction of propagation of these waves. Time difference of arrival of these waves at each elements of the antenna is measured to determine AoA. Multiple signal classification (MUSIC) algorithm is the famous one on this category.

In proposed scenario filed sensors are not equipped with GPS unit, hence GPS based localization schemes cannot be considered. GPS free schemes are also not suitable as they required to install large-size, heavy wait and energy-consuming physical antenna system to locate scattered sensor nodes in a large geographical area which is not possible to install on a small size UAV.

\section{Proposed system}

In this research, a special virtual phased array (VPA) antenna system is proposed which will replaces the physical antenna array. Following shortcomings of the classic localization system is improved in our proposed VPA antenna: 
1. Size and weight: As discussed earlier, a massive array of multiple antennas decreases speed, flight time, payload capacity, and agility of a small size UAV. In this proposed system, the established VPA replaces the physical antenna and its limitations.

2. Throughput: When UAVs are used for data collection the biggest issue is high round trip time (RTT) and very poor throughput. The UAV equipped VPA will help to overcome this issues by adapting localization attributes through varying its speed and height.

3. Capabilities: Steering ability of a physical antenna cannot/difficult to alter during operation while, in a virtual antenna, it is a trade-off between accuracy and speed.

4. Detectable targets: In VPA number of antenna elements participating in localization process is virtually adjustable, this property enable it to adjust total number of detectible targets at a time.

5. Multi frequencies: As inter-element spacing is not easily re adjusted in a physical onboard antenna, so it is not possible to operate at multiple frequencies. However, this is possible with the VPA.

6. Others: Typical issues with a physical antenna, such as inter-channel phases, mutual coupling, and current gains, between array elements do not exist in our proposed VPA.

In our scenario, all field sensor nodes are emitting a narrowband signal that is sensed by an antenna (VPA) attached with the UAV. This single antenna virtually makes a $M$ physical antenna elements by exploiting the mobility of the UAV. Continuous snapshots of the data are taken by UAV at a fixed interval of time and distance. $M$ data Snapshots are manipulated in such a way that VPA produces same result as a physical antenna can do with $M$ elements, exhibit is shown in Figure 1.

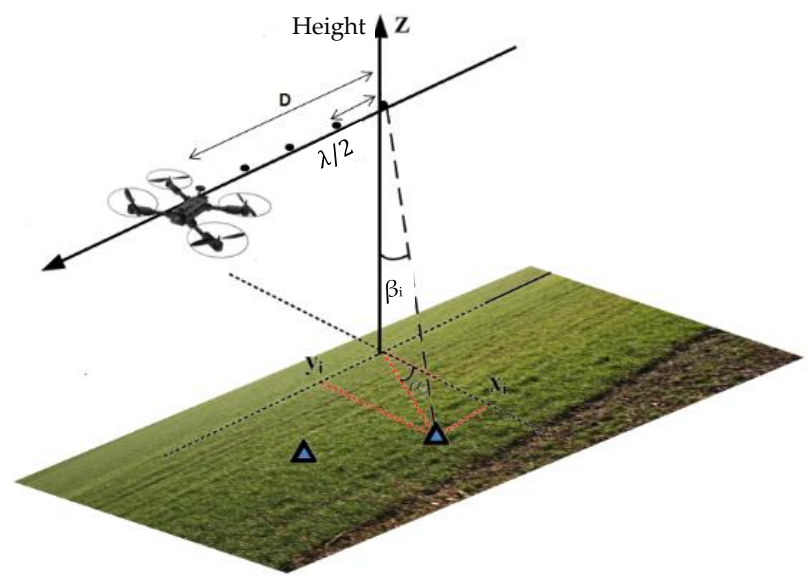

Figure 1. Localization of ground target node by UAV

\subsection{Uniform linear antenna for localization}

A set of antennas (antenna array) is used to find the accurate AoA of a signal, which is then further processed to locate a target node. This type of antenna formation is made either by a static array composed of a number of elements or a single rotating antenna to form a radar. There is a third exceptional case in which multicell static radar is used in aircrafts. This array antenna can detect many parameters (other than AoA) of an incoming signal, including arrival time, incident angle, frequency, range, and polarization. An array antenna can detect the AoA of received signals from multiple target nodes with high resolution in terms of angle (elevation $\Phi$ and azimuth $\theta$ ). These received signals are considered as plane wave incident rays of any type narrowband or wideband.

AoA estimation accuracy (resolution) varies with array formation geometry (how the antenna elements are organized). Some of the famous antenna array geometries are uniform rectangular array, uniform linear array (ULA), L-shaped array, uniform circular array (UCA), concentric circular array, displaced sensor arrays, Vshaped array, Y-shaped arrays, and parallel linear arrays for details, see [38], [39], [40]. ULA [41] is the simplest antenna formation and requires the least computation. UCA, as presented in [38], is more complex than ULA but provides consistent performance in all azimuth directions in 2D plan.

Narrower beam of radio waves with higher gain/directivity can only be possible to attain with an array of antenna and could not be achieved with one element. In general, the more antenna elements, the higher the 
gain and narrower the beam. However, let us consider a ULA antenna having $M$ elements where $M=$ $\left(m_{1}, m_{2}, \ldots, m_{M}\right)$, operating at radio frequency $f_{0}$ of wavelength $\lambda$, as shown in Figure 2.

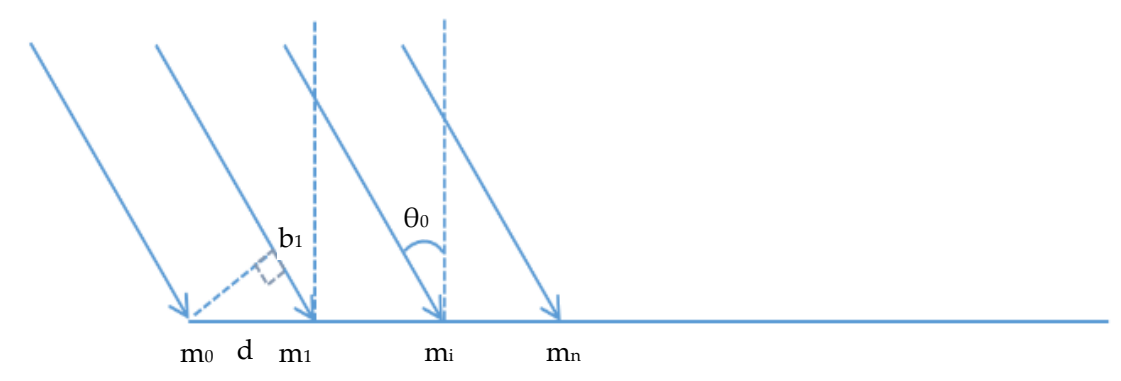

Figure 2. Direction of arrival measurement by ULA

$$
c=\lambda f_{0}
$$

Let the separation between two adjacent antenna elements is $d=\frac{\lambda}{2}=\overline{m_{0} m_{1}}$, where $m_{1}$ the first antenna element is and $m_{i}$ be the $i$ th antenna element. In this case, it can be written as:

$$
\overline{m_{1} b_{1}}=l=d \sin \left(\theta_{0}\right) \text {. }
$$

Let signal received by the first antenna is $s(t)$ taken as reference:

$$
x_{1}(t)=s(t)=\cos \left(\omega_{0} t\right)=\cos \left(2 \pi f_{0} t\right) .
$$

The $2^{\text {nd }}$ antenna element $x_{2}(t)$ will receive the same signal is with a delayed of $\tau$ :

$$
\begin{gathered}
x_{2}(t)=s(t-\tau)=\cos \left(\omega_{0}(t-\tau)\right) \\
=\cos \left(\omega_{0} t-\omega_{0} \tau\right) .
\end{gathered}
$$

The delay $\tau$, as per Eq. (2), can be written as

$$
\tau=\frac{l}{c}=d \frac{\sin \left(\theta_{0}\right)}{c} .
$$

The gained phase difference between adjacent array elements due to $\tau$ becomes

$$
\varphi_{0}=\omega_{0} d \frac{\sin \left(\theta_{0}\right)}{c} \text {. }
$$

Similarly, phase difference at the $i^{\text {th }}$ antenna element is given by:

For a complex signal,

$$
\begin{gathered}
\Phi_{i}=(i-1) \omega_{0} \frac{d \sin \left(\theta_{0}\right)}{c}=(i-1) 2 \pi \frac{f_{0}}{c} d \sin \left(\theta_{0}\right) \\
=(i-1) \frac{2 \pi d}{\lambda} \sin \left(\theta_{0}\right)
\end{gathered}
$$

$$
x_{i}(t)=s(t) e^{-j \Phi_{i}}
$$

Finally, $\mathrm{A}(\theta)$ is the steering vector of the incident signal with $M$ antenna elements is derived as

$$
\mathrm{A}(\theta)=\left(\begin{array}{c}
1 \\
e^{-j \theta_{0}} \\
e^{-2 j \theta_{0}} \\
\vdots \\
e^{e^{-j}(\mathrm{M}-1) \theta_{0}}
\end{array}\right) .
$$

\subsection{Multiple sources in ULA}

If there are $N$ independent sources then resultant signal of the $m^{\text {th }}$ antenna element is provided by the equation

$$
x_{m}(t)=\sum_{k=1}^{N} s_{k}(t) e^{-j(m-1) \varphi_{k}}+b_{m}(t)
$$

Where, incident angle of the $K^{t h}$ source is $\varphi_{k}=\frac{2 \pi d}{\lambda} \sin \left(\theta_{k}\right)$, and $b_{m}(t)=A W G N$ (Additive White Gaussian Noise). In this illustration, the observation vector can be written as 


$$
X(t)=\left(\begin{array}{c}
x_{1}(t) \\
\vdots \\
x_{M}(t)
\end{array}\right)=\left(\begin{array}{cccc}
1 & 1 & \cdots & 1 \\
e^{-j \varphi_{1}} & e^{-j \varphi_{2}} & & e^{-j \varphi_{N}} \\
\vdots & \vdots & & \vdots \\
e^{-j(M-1) \varphi_{1}} & e^{-j(M-1) \varphi_{2}} & \cdots & e^{-j(M-1) \varphi_{N}}
\end{array}\right)\left(\begin{array}{c}
s_{1}(t) \\
\vdots \\
s_{N}(t)
\end{array}\right)+\left(\begin{array}{c}
b_{1}(t) \\
\vdots \\
b_{M}(t)
\end{array}\right)
$$

The observation vector in this case can be developed as follows

$$
X(t)=\left(\begin{array}{lll}
A_{1}\left(\theta_{1}\right) & A_{2}\left(\theta_{2}\right) \ldots . A_{M-1}\left(\theta_{N}\right)
\end{array}\right)\left(\begin{array}{c}
s_{1}(t) \\
\vdots \\
s_{N}(t)
\end{array}\right)+\left(\begin{array}{c}
b_{1}(t) \\
\vdots \\
b_{M}(t)
\end{array}\right)=A S+B,
$$

where $X=\left[x_{1}(t), x_{2}(t), \ldots, x_{M}(t)\right]^{T} ; S=\left[s_{1}(t), s_{2}(t), \ldots, s_{N}(t)\right]^{T} ; B=\left[b_{1}(t), b_{2}(t), \ldots, b_{M}(t)\right]^{T}$; and $A=\left[A_{1}\left(\theta_{1}\right), A_{2}\left(\theta_{2}\right), \ldots, A_{M-1}\left(\theta_{N}\right)\right]$.

\subsection{Multiple signal classification}

A famous technique to find AoA of incoming signals is MUSIC published in [42]. In this technique observed data by an array of antenna is represented in covariance matrix then signal and noise subspaces are separated by and eigenvalue decomposition (EVD) method. Then two orthogonal subspaces are utilized to constitute a spectrum function. Finally, the AoAs of signals are revealed by maximizing the spectral criterion.

By applying an EVD on $R_{X}$, it can be proved that

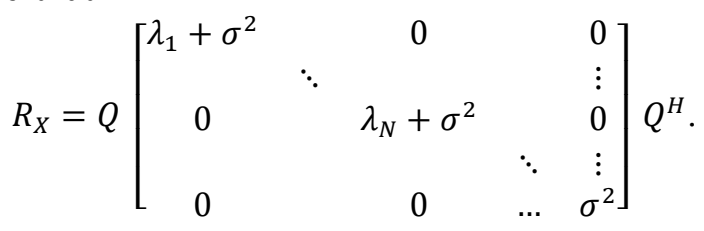

Obtained $Q$ matrix can be divided into two matrices, $Q_{S}$ and $Q_{n}$ by using above equation, such that $Q_{S}$ is the $M \times N$ matrix and $Q_{n}$ is $M \times(M-N)$ matrix. In this case, MUSIC becomes equal to

MUSIC algorithm:

$$
\max P_{\text {MUSIC }}(\theta)=\frac{1}{\sum_{k=1}^{N-M}\left(A^{H}(\theta) g_{k}\right)^{2}}=\frac{1}{\left\|Q_{n}^{H} A(\theta)\right\|^{2}} \text {. }
$$

1) Estimate covariance matrix: $\hat{R}_{X}=\frac{1}{K} \sum_{1=1}^{K} X(i) X^{H}(i) ; K=$ number of samples

2) Evaluate EVD of $\hat{R}_{X} \Rightarrow \hat{R}_{X}=Q \wedge Q^{H}$

3) Separate $Q$ into two matrixes: $Q=\left(\begin{array}{ll}Q_{S} & Q_{n}\end{array}\right)$

4) Plot a function of $\theta:\left\|Q_{n}^{H} A(\theta)\right\|^{2}$

5) The $M$ larger peaks of PMUsic represent the AoA

The data model is the key point of the MUSIC algorithm. If $N$ and $M$ are the number of, signal sources and antenna elements respectively such that $M>N$. The output signal at any time $t$ for array element $m$, is given by

$$
x_{m}(t)=\sum_{k=1}^{N} a_{m}\left(\theta_{k}\right) s_{k}(t)+n_{m}(t),
$$

Where, $S_{k}(t)$ is a narrowband signal, $n_{m}(t)$ is the white noise component, and $a_{m}$ is the response function of the $m$ array element of the $K^{\text {th }}$ signal source, and. Eq. (13) can be written in matrix form, as follows:

where, $X=\left[x_{1}(t), x_{2}(t), \ldots, x_{m}(t)\right]^{T}$,

$$
X=A S+N \text {, }
$$

$s=\left[s_{1}(t), s_{2}(t), \ldots, s_{D}(t)\right]^{T}$, and the steering vector is given by $A=\left[a\left(\theta_{1}\right), a\left(\theta_{2}\right), \ldots, a\left(\theta_{D}\right)\right]^{T}$.

This classical MUSIC algorithm perform only in case of fixed length and number of elements of an antenna. Furthermore it is very sensitive to sensor position, gain and phase errors and needs careful calibration. While in case of our scenario all the samples of data are not collected at once by all antenna elements, and this property modifies the signal features more than usual. In this regard classical MUSIC algorithm is not usable in our scenario in its current state. To accommodate these additional signal deviations, some modifications and additions are suggested MUSIC algorithm to upgrade it for proposed virtual antenna array system. 


\section{Proposed virtual antenna array architecture}

A VPA carrying UAV, named as synthetic aperture UAV (SA-UAV) is used for node detection and localization. In proposed scheme, AOA is estimated using collected data in at a certain point in response of incoming signals of different phases at different times. Assume the SA-UAV is locating $N$ target nodes emitting narrowband signals dispersed in a crop field. First of all we assumed transmission channel is quasi-stationary while taking one snapshot of data (less than a second), which is practical. Let's SA-UAV acquires snapshots at $M$ different places where $M=N+1$. Each snapshot is taken at a fixed period of $\Delta t$ and is consists of $R_{\text {over }}$ samples. All the received signals (coming from target nodes) is considered a plane wave as the SA-UAV satisfies the far-field condition of the frequency while flying by maintaining its height. Working with VPA is more complex than a physical ULA, and special care and treatment is required to collect and process data samples as incoming signals changing its characteristics with varying position. Classical localization system (e.g., the MUSIC algorithm) is upgraded by adding new modules in the systems such as calibration, rectification, and adjustment as shown in Figure 3.

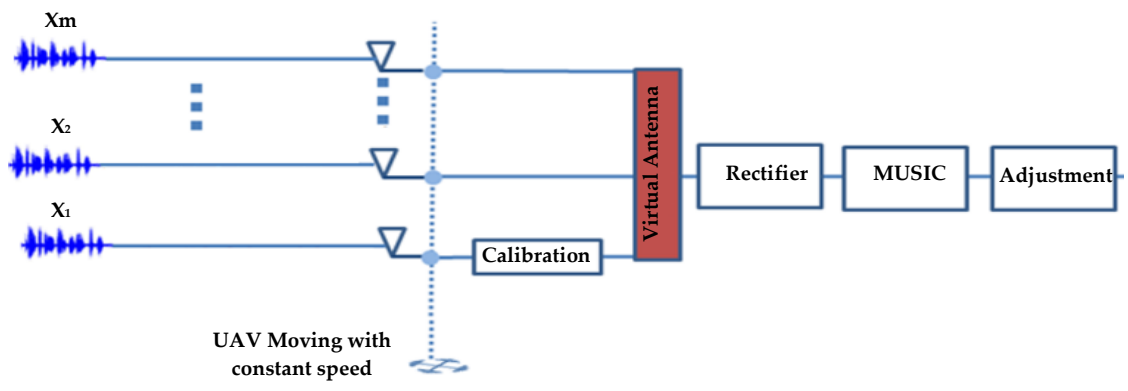

Figure 3. Proposed virtual phased array antenna system and its components

Let $x_{i}(t)$ is $x$ independent signals collected by VPA at time $t$ at in $i$ th snapshot. Same signal monitor by two adjacent antenna elements has fixed phase deviation because each element is separated by $\lambda / 2$ (half of the frequency) in physical antenna. In VPA, there is an extra deviation due to factors such as the geometrical variation of the antenna, signal-to-noise ratio (SNR), and desynchronization clocks (Sensor node and UAV) cause. Desynchronized cock of devices causes phase difference $F_{\text {offset }}$ between two consecutive samples. This desynchronization is estimated in introduced module called calibration in the start of the system in first sample pair then it is considered as constant throughput the proceeding samples. Virtual antenna module (VAM) collects all the samples and accumulate whole the data. VAM forms steering vector based on each signal response and forward it to the rectifier module (RM). RM module rectify the entire data-set by fixing $F_{\text {offset }}$ and create a covariance matrix $R_{A S}$. After rectification, data is ready to process by classical MUSIC algorithm. This classical MUSIC algorithm gives is an angle $\theta$ as an output that is once again corrected by Adjustment module. Proposed modules are explained in detail in the following section.

\subsection{Geometrical deviation}

As all the samples of signals are not taken at once by each antenna elements (as in case of physical array antenna) in this case signal characteristics are varying for every sample due to the continuously moving position of the antenna. Therefore, first of all the geometrical variation of the antenna must be defined. If $N$ sensor nodes $\left(n_{1}, n_{2}, \ldots, n_{N}\right)$ are installed, then node $n_{i}$ transmutes signal which makes the angle $\left(\theta_{1}^{n_{1}}, \theta_{2}^{n_{1}}, \ldots, \theta_{M}^{n_{1}}\right)$, with virtual antenna having $M$ elements (see Figure 4). The UAV flight is taken as a straight path where $Y$ axis is taken as constant and the $X$ axis is considered linearly corresponding to the trajectory. 


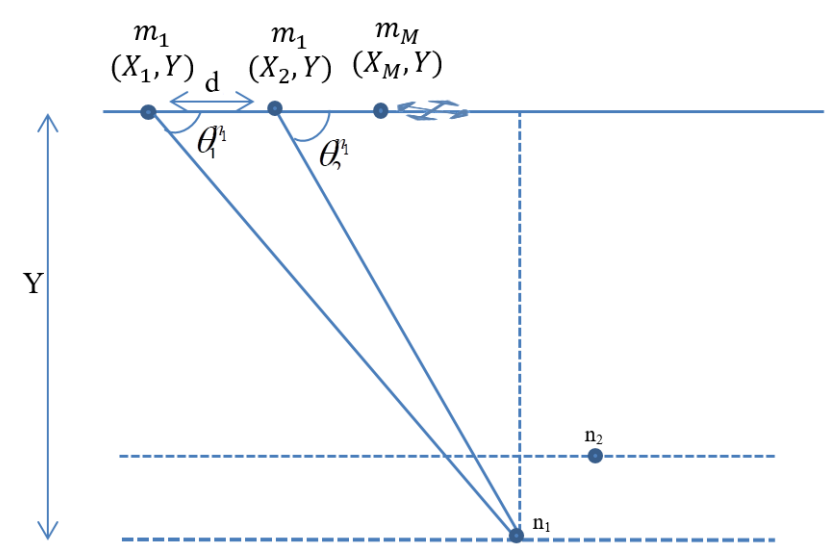

Figure 4. The geometric angle deviation for the VPA antenna

As exhibited in Figure 4, the abscissa of UAV is $X_{k}$ at time $t_{k}$, where $1<k \leq M$ while the ordinate (Y axis) is the constant for all nodes. $N$ Target nodes $\left(n_{1}, n_{2} . . n_{N}\right)$ where $1 \leq i \leq(N=M-1)$ are installed in a field. The $M$ is the number of data snapshots taken by the SA-UAV after a fixed distance of

$$
d=X_{k+1}-X_{k}=c_{u} \Delta t
$$

If the UAV takes two successive snapshots in time $\Delta t$ covers a distance $d$ with a speed $c_{u}$ then the angle of sensor node $n_{i}$ with first antenna element $X_{1}$ is determined by

$$
\tan \theta_{1}^{n_{1}}=\frac{X_{1}}{Y}
$$

If the reference is first snapshot, then the angle of arrival for the next snapshot is determined as

$$
\tan \theta_{2}^{n_{1}}=\frac{X_{1}-d}{Y}
$$

As the flight of UAV is linear, it is written as

$$
\theta_{k}^{n_{i}}=\theta^{n_{i}}\left(t_{k}\right)=\tan ^{-1}\left(\frac{X_{k}-c_{u} t_{k}}{Y}\right)
$$

As $\Delta t=\frac{\lambda}{2 c_{u}}$ seconds (where $d=\lambda / 2$ is the inter element spacing). Then steering vector, or an array manifold, for the node $i$ of angle $\theta$ is $M \times 1$. The observation matrix is derived as

$$
A(\theta)=\left[a_{1}\left(t_{1}\right), a_{2}\left(t_{2}\right), \ldots a_{M}\left(t_{N}\right)\right]^{T}
$$

VPA can model the physical antenna array if $t_{i}$ and the observations $A(\theta)$ are known. As the data sampling frequency is very higher as compare to the snapshotting frequency which is related to the speed of the UAV. If $\mathrm{UAV}$ is maintains a constant speed $c_{u}$ during taking one snapshot (antenna element), then it is considered as uniform VPA antenna of $M$ elements. If first snapshot is taken as a reference, and the steering vector is derived as

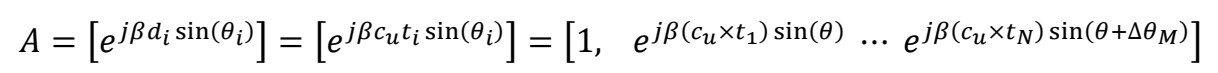

Where, $i=1,2, \ldots, M, \beta=\frac{2 \pi}{\lambda}, \lambda$ is the wavelength, the inter-snapshot spacing is $d=\lambda / 2$, and $\theta$ is the AoA of the sensor. To find a target UAV takes first snapshots at time $t_{1}=0$ and at time $t_{m}$ it approaches the $M^{\text {th }}$ snapshot. $\Delta \theta$ is the rate of change of the incident angle of the signal due to movement of the UAV in between each snapshot. A key advantage of our developed system is that it can operate at different frequencies as it can change interelement spacing and adjust frequency of samplings during UAV operation if required. Two antenna configuration with different frequencies are described below.

\subsection{Example 1: Long-range localization}

For long range communication a UHF frequency band is used. If field sensors are operating at $433 \mathrm{MHz}$ frequency to cover a long distance with very low power. Localization of such nodes are possible with VPA and it will have following specifications:

Operational frequency

$f_{1}=433 \mathrm{MHz} \quad \lambda_{1}=\frac{3.10^{8}}{4.33 \cdot 10^{8}}=0.6928 \mathrm{~m} \sim 0.7 \mathrm{~m} ;$ 
Speed of UAV

$$
c_{u}=25 \mathrm{~km} / \mathrm{h}=6.944 \mathrm{~m} / \mathrm{s} \sim 7 \mathrm{~m} / \mathrm{s}
$$

UAV will take a sample after every

$$
\Delta t=\lambda 1 /\left(2 C_{u}\right)=0.05 s=50 m s ;
$$

As defined in reference [43] the cross-range resolution of an antenna is $\Delta \theta=\frac{\lambda 1}{L_{f 1}}$ radians. Radian is converted to degree as

where, $L_{f 1}$ is the length of the antenna with frequency $f_{1}$.

$$
\Delta \theta=57 \frac{\lambda 1}{L_{f 1}}
$$

If the antenna resolution $\Delta \theta_{3 d B}=6^{\circ}$ (3dB beam width) is required, then the antenna length should be

$$
L_{f 1}=57 \frac{\lambda 1}{6^{\circ}}=6.65 \mathrm{~m} \text {. }
$$

The spacing between two virtual antenna elements is $\lambda / 2=0.35 \mathrm{~m}$, and the number of snapshots (antenna elements) to complete VPA antenna is

$$
M=\left\lceil\frac{L_{f 1}}{\lambda / 2}\right\rceil=20
$$

The time of observation for all snapshot to complete one set of antenna is as follows:

$$
\text { Tobs }=L_{f 1} / c_{u}=6,65 / 7 \sim 0.95 \mathrm{sec} .
$$

\subsection{Example 2; Short-range localization}

If field sensor nodes are operating at $2.4 \mathrm{GHz}$ frequency for short range and huge data communication. Localization if such nodes are possible if antenna have following specifications

Operating frequency $\quad f_{2}=2.4 \mathrm{GHz} ; \quad \lambda_{2}=\frac{3 \times 10^{8}}{24 \times 10^{8}}=0.125 \mathrm{~m}$

UAV will take a sample after every $\Delta t=\frac{\lambda_{2}}{2 c_{u}} \sim 0.009 \mathrm{~s}=9 \mathrm{~ms}$

If the minimum required resolution is $\Delta \theta_{3 d B}=6$

The antenna length will be $L_{f 2}=57 \frac{\lambda_{2}}{6^{\circ}}=1.1875 \mathrm{~m}$

The number of antenna elements (number of snapshots) for a complete virtual array will be $M=20$.

\subsection{Sampling module}

For $M$ snapshots total $R_{\text {over }} \times M$ samples are required to achieve precision means $R_{\text {over }}$ samples are collected between every two snapshots $m_{k}$ and $m_{k+1}$. Over sampling of the analog-to-digital converter (ADC) used in VPA also effects this factor $R_{\text {over }}$ and performance of VPA. If $F_{A D C}$ (in $\mathrm{Hz}$ ) is the measured ADC sampling frequency, then oversampling for one snapshot is

where, \lfloor] stand for integer part.

$$
R_{\text {over }}=\left\lfloor F_{A D C} \Delta t\right\rfloor
$$

If VPA antenna is operating at $F_{\text {radio }}=433 \mathrm{MHz}$ frequency for communication and a standard ADC converter (e.g., a voltage-controlled crystal oscillator) installed. As per data sheet it has an accuracy of $10 \mathrm{ppm}<$ Frequency accuracy $<50 \mathrm{ppm} \quad(\mathrm{ppm}=$ part per million $)$, then it generates a frequency offset of

$$
4.33 \mathrm{KHz}<\Delta F<21.65 \mathrm{KHz} \text {. }
$$

By sampling the period $\frac{1}{F_{A D C}}$, the frequency offset causes a rotation of phase according to $\Delta \phi_{\text {offset }}=\frac{\Delta F}{F_{A D C}}$

$$
0.02165 \times 2 \pi<\left|\Delta \phi_{\text {offset }}\right|<0.10825 \times 2 \pi
$$


It means between two consecutive samples $\frac{2 \pi}{10}$ extra phase rotation will exists in worst case scenario (poorest accuracy result). This is because VPA samples are independent (taken one-by-one) in contrast of physical antenna array. This $\Delta \phi_{\text {offset }}$ phase difference is other then what is expected which must be fixed.

\subsection{Calibration module}

As discussed in previous section phase difference between two successive data samples is caused by ADC oversampling. Before processing the signals data array the compensation for this phase rotation between two consecutive samples need to estimate.

To fix this issue without a loss of generality, assume the scenario with a singular source, and let $s(k)$ represents the $\mathrm{k}^{\text {th }}$ sample of that source received by the antenna at $k$ discrete time.

$$
s(k)=e^{-j 2 \pi \Delta F_{\text {total }} \frac{k}{F_{A D C}}}
$$

Where $k=1,2, \ldots, R_{\text {over }}$. The frequency offset difference of the two clocks (UAV, and the node) is $\Delta F_{\text {total }}$

$$
\Delta F_{\text {total }}=F_{U A V}-F_{\text {node }}
$$

$\Delta F_{\text {total }}$ Causes the angle of rotation between two samples as

$$
\Delta \Phi=\Phi_{k}-\Phi_{k-1}=\frac{2 \pi \Delta F_{\text {total }} k}{F_{A D C}}-\frac{2 \pi \Delta F_{\text {total }}(k-1)}{F_{A D C}}
$$

From (24) and (25), the frequency offset the difference $\Delta F_{\text {total }}$ can be written as

$$
\Delta F_{\text {total }} \approx \frac{\Delta \Phi R_{\text {over }}}{2 \pi \Delta t}
$$

During whole observation time $\Delta F_{\text {total }}$ is constant. For a unitary observation time of $\Delta t$ (between two samples of the VPA), approximated as

$$
\Delta F_{\text {total }} \approx \widetilde{\Delta \mathrm{F}}_{\text {offset }} \cong \frac{\sum_{k=2}^{R \text { over }}\left(\Phi_{k}-\Phi_{k-1}\right)}{R_{\text {over }}-1}
$$

\subsection{Rectification module}

The rectification module is introduced to fix phase offset by applying compensation of $\widetilde{\Delta \mathrm{F}}_{\text {offset }}$.

$$
S_{r}(k)=e^{-j 2 \pi \widetilde{\Delta F}} \text { offset } \frac{k}{F_{A D C}} S(k)
$$

\subsection{Adjustment module}

In this scenario, UAV covered distance and its speed defines the incident $\theta$ of each signal sample capture by VPA. The final output $\theta$ value must be adjusted as per following Figure 5 .

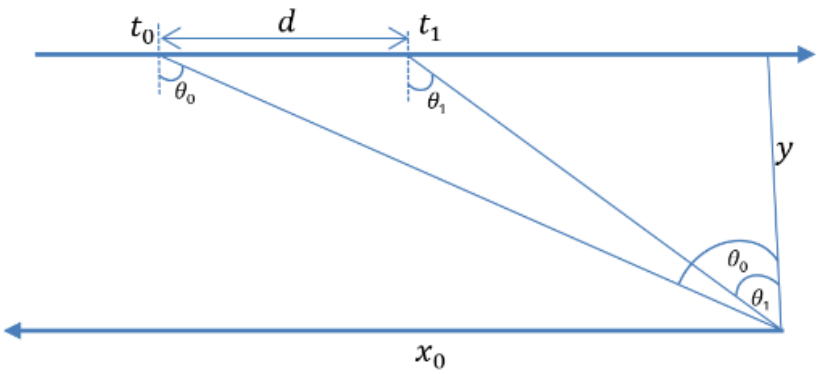

Figure 5. The angle correction by adjustment module of the virtual phased array antenna

As in Figure 5, let $i$ th snapshot is taken when incident angle is $\theta_{i}$ and the horizontal distance between the UAV and node is $x_{i}$ while the height of UAV $y$ is constant. In this case, it is concluded that $x_{i}=x_{i+1}+d$ and $\theta_{i}=$ $\tan ^{-1}\left(\frac{x_{i}}{y}\right)$, where $d$ the distance travels between two consecutive snapshots. If UAV is moving with content speed $c_{u}$ and time between two snapshots is $\Delta t$ then $d=c_{u} \Delta t$. Using a first-order approximation, the adjustment is initially written as 
Therefore, the total adjustment $\Delta \theta$ should be

$$
\begin{gathered}
\Delta \theta=\theta_{i}-\theta_{i+1}=\tan ^{-1}\left(\frac{x_{i}}{y}\right)-\tan ^{-1}\left(\frac{x_{i}+1}{y}\right) \\
\approx \frac{x_{i}}{y}-\frac{x_{i}-d}{y}=\frac{c_{u} \Delta t}{y}
\end{gathered}
$$

$$
\Delta \theta_{T}=M \Delta \theta
$$

\subsection{Effect of non-linear movement of UAV on VPA antenna}

The virtual antenna array is developed on an assumption that the UAV is flying in a straight path at a constant speed and height (for at least one snapshot). All the snapshots of data are captured at equal intervals of time. The UAV takes its first snapshot $m_{1}\left(X_{1}, Y_{1}\right)$ at Point $\mathbb{A}$ and a next snapshot $m_{2}\left(X_{2}, Y_{2}\right)$ at point $\mathbb{B}$ as shown in Figure 6.

It is assumed that the UAV is operating in a straight path for a time period long enough to take one snapshot of data (length of one antenna element), which is less than a second. If this assumption is not true, consider the case in which air pressure or GPS inaccuracies may deflect the UAV from a straight path, as shown in Figure 6. At Point $\mathbb{B}$, the UAV deflects by angle $\alpha$ from a straight path, and it takes the second snapshot at Point $\mathbb{C}$. As snapshots are recorded after fixed intervals of time, the distance between the two points (Al and $\mathbb{C})$ is still $\lambda / 2$.

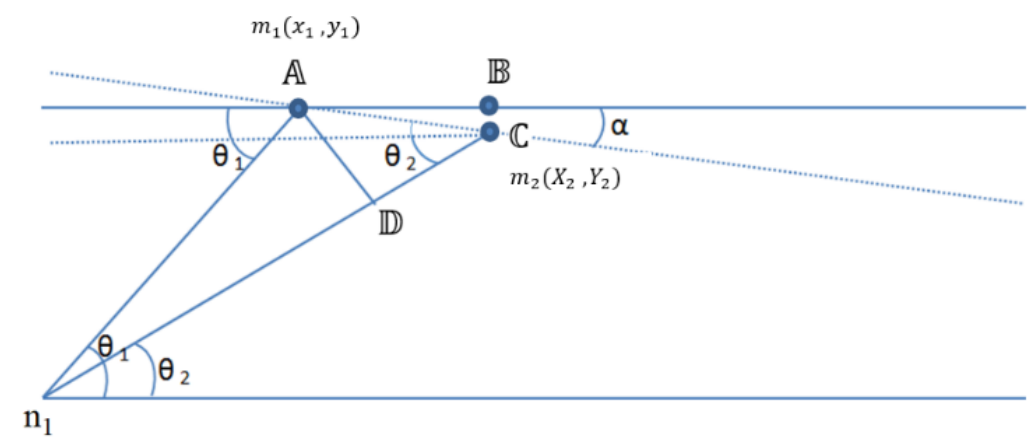

Figure 6. UAV non-linear movement and angle of arrival estimation

If letting $D_{i}=$ distance from $m_{1}$ to the point $m_{i}=\sqrt{x_{i}^{2}+y_{i}^{2}}$, the transmission time required to cover the distance $D_{i}$ is

$$
\tau_{i}=\frac{D_{i}}{c}
$$

where $c=$ speed of light. Let us consider a relative delay, i.e., the antenna time starts when the first signal $m_{1}$ is received.

If $\mathbb{A}=\left(x_{1}, y_{1}\right)$ is taken as a position of the first point and $\mathbb{C}=\left(x_{2}, y_{2}\right)$ is the position of second point which is deflected by angle $\alpha$ from the straight path where point $\mathbb{B}=(\mathrm{x}, \mathrm{y})$ resides. In this case, it can be proved that

$$
\begin{gathered}
\tan \alpha=\frac{\overline{\mathrm{BC}}}{\overline{\overline{\mathrm{AC}}}}=\frac{y_{1}-y_{2}}{x_{2}-x_{1}} \\
\tan \theta_{i}=\frac{y_{i}}{x_{i}} .
\end{gathered}
$$

The actual delay between the consecutive antenna elements is given by

$$
\tau=\frac{D_{2}-D_{1}}{c}=\frac{\sqrt{x_{2}^{2}+y^{2}}-\sqrt{x_{1}^{2}+y^{2}}}{c} .
$$

As $d=\overline{\mathrm{AC}}=\overline{m_{1} m_{2}}$, the distance between $\overline{\mathrm{BC}}$ is

$$
\overline{\mathbb{C D}}=d \cos \left(\theta_{2}+\alpha\right) \text {, }
$$

Where $y_{1}-y_{2}=d \sin \alpha$ and $x_{2}-x_{1}=d \cos \alpha$

In this case, it can be written as

$$
\tau C=\sqrt{\left(x_{1}+d \cos \alpha\right)^{2}+\left(y_{1}-d \sin \alpha\right)^{2}}-\sqrt{x_{1}{ }^{2}+y_{2}{ }^{2}}
$$

Let $D$ be the distance between sensor node and UAV: 
Assume that $D \gg d$ :

$$
D=\sqrt{x_{1}^{2}+y_{1}^{2}}
$$

$$
\begin{aligned}
\tau C & \left.=\sqrt{D^{2}+d^{2}+2 d\left(x_{1} \cos \alpha-y_{1} \sin \alpha\right.}\right)-D \\
\tau C & \cong D \sqrt{1+\left(\frac{d}{D}\right)^{2}+\frac{2 d}{D} \cos (\alpha+\theta)}-D \\
& \cong D \sqrt{1+\frac{2 d}{D} \cos (\alpha+\theta)-D} \\
& \cong D\left(1+\frac{d}{D} \cos (\alpha+\theta)\right)-D \\
& \cong d \cos (\alpha+\theta) .
\end{aligned}
$$

The $\alpha$ is considered as negligible because the antenna length and time required to collect all of the data samples is very small.

\section{Simulation study and result analysis}

The proposed VPA antenna system is evaluated by a series of experiments conducted by simulation model created in MATLAB. A simulation model is created in which a $433 \mathrm{MHz}$ solo antenna is installed in a UAV that moves with a constant speed of $20 \mathrm{~m} / \mathrm{s}$. UAV is given a target to locate three target nodes placed in an open field emitting narrowband signals $a(\theta)$ with azimuth angles of $-40^{\circ},-10^{\circ}$ and $+20^{\circ}$. The elevation angle is taken to be 0 for all nodes.

Separate simulation test cases are created to find the directions of targets nodes with and without the proposed system and results are shown in Figure 7. Results shows that AoAs of these three target nodes are measured accurately by the UAV using our proposed system. The simulation results of classical MUSIC algorithm without modification is shown in black curve which clearly depict that it does not work well under given circumstances. The red curve shows a successful MUSIC process after incorporating the developed modules, including the calibration, rectification and adjustment. Means using proposed VPA all three targets are located accurately as we can do in physical antenna array system.

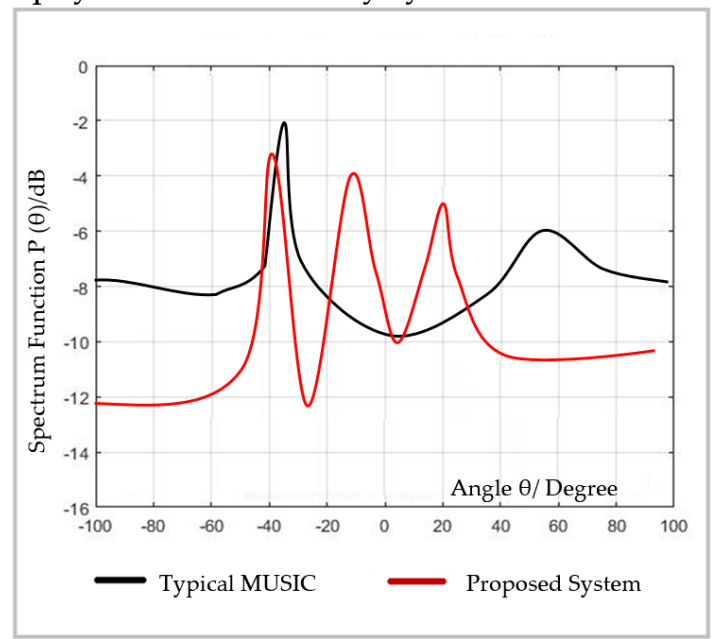

Figure 7. Performance of classical MUSIC algorithm vs proposed system

The factor that strongly effects the system performance is SNR. Increasing SNR may degrade the system. The performance of the system was also evaluated against different SNR values and results are shown in Figure 8 . Location detection accuracy of three angles -40 -10 and 20 degree was tested against increasing SNR values and found that the proposed system works satisfactory with a low value of SNR up to $4 \mathrm{~dB}$. 


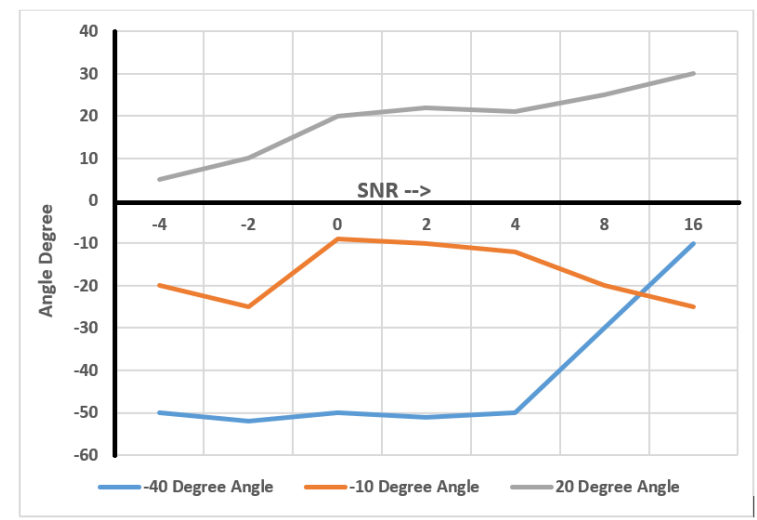

Figure 8. Performance evaluation of proposed AoA estimation under increasing the SNR

As we proved in section 3.4 if we are using standard ADC convertor (having accuracy $10 \mathrm{ppm}$ to $50 \mathrm{ppm}$ ) to sample radio signal of frequency $433 \mathrm{MHz}$ then each consecutive sample will have $2 \pi / 10$ phase different due to movement of UAV. If each signal is represented by different color then Figure 9 shows the picture of received signals without rectification, and it is observed that every new sample of same signal is deviated by 36 degree. Means each received signal takes ten rotations per sample which causing major deviations in the AoA estimation.

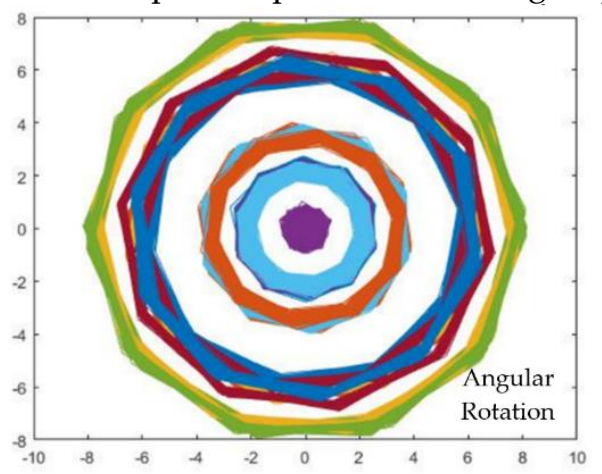

Figure 9. The received signal without rectification

This signal rotation issue is fixed by proposed treatment called rectification. The treated signal is shown in Figure 10, in which the rotation of phases due to ADC oversampling is fixed successfully. One can see a focused signal is received throughout the sampling process.

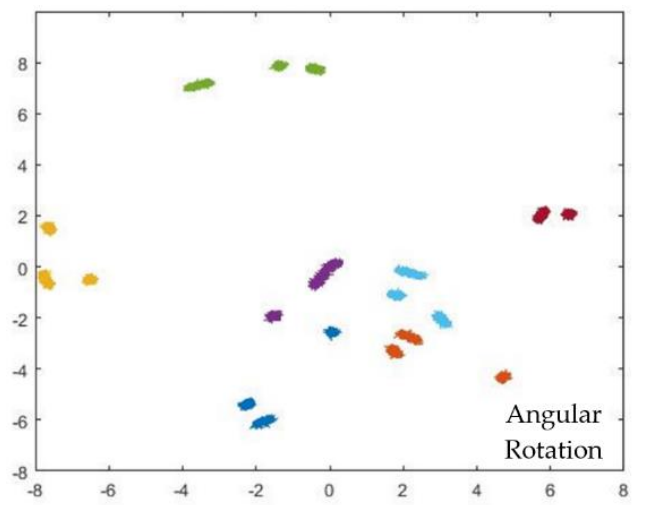

Figure 10. Treated signal with rectification module

We also tested our system when angle is close to 0 or transforming from negative to positive. An angle of +-2 degree is taken as target and in the Figure 11, it is shown that it measured accurately.

All simulation outcomes provided above supports our claim that the physical antenna can be replaced by virtual phase array antenna effectively without degradation of results. VPA will add many benefits including cost-effectiveness, light-weightiness, ease of use, flexibility, adaptability, and energy efficiency. 


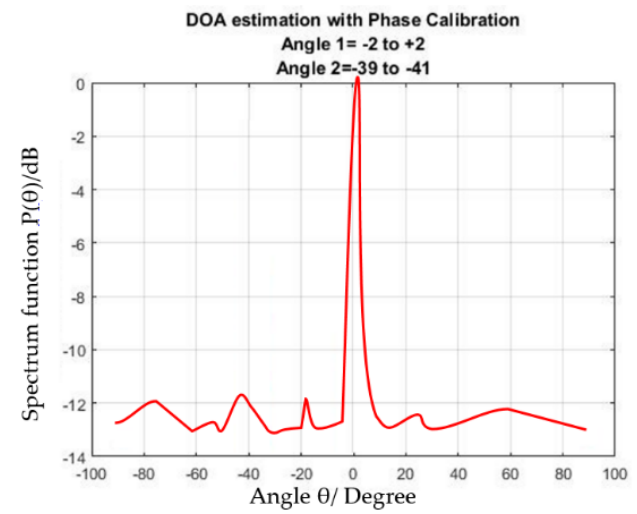

Figure 11. Accuracy of the system with varying of angle from negative to positive

\section{Conclusion}

A virtual phased array antenna system is proposed for the replacement of large size physical linear array antenna. The motivation behind the proposed system is that I can be installed on a small small-sized UAVs. In this VPA system, the AoAs of incoming narrowband signals are captured by a SA-UAV at a fixed interval of time to model the observation matrix as obtained in physical antenna elements. The observed data is then treated with calibration, rectification, and adjustment modules to negate the effect of signal variation due to antenna movement. It has been proved mathematically and verified by the simulation model that a solo antenna mounted on a moving UAV can act as a physical antenna of multiple elements. The benefits, such as being lightweight, small size and energy-efficient and possessing multi-frequency use, adaptable precision and better throughput make it more suitable for drone use.

\section{Data availability (Author elects to not share data)}

Research data are not shared, however the simulation videos are available on YouTube and can be accessed through the links given bellow. The first video shows how UAV and target get connected and complete localization process.

The second video is showing how multiple target nodes are identified by UAV and share information after finding their location. Matlab simulation of localization and cluster formation of target nodes by UAV is provided in the video 3.

1. https://www.youtube.com/watch?v=xzf4SkhDteE\&t=14s

2. https://www.youtube.com/watch? $\mathrm{v}=\mathrm{OXtNn} 9411 \times A \& \mathrm{~A}=50 \mathrm{~s}$

3. https://www.youtube.com/watch? $=\mathrm{zVM} 42 \mathrm{i} 9 \mathrm{BQFY}$

\section{Acknowledgment}

This work was supported in part by the SNCS Research Center and in part by the Deanship of Scientific Research at the University of, Tabuk, Tabuk, Saudi Arabia, under project No. S-1441-0075.

\section{References}

[1] H. González-Jorge, J. Martínez-Sánchez, M. Bueno, and and P. Arias, "Unmanned Aerial Systems for Civil Applications: A Review,” Drones, vol. 1, no. 1, p. 2, Jul. 2017.

[2] G. D. Friedrich, "Applications of military and non-military Unmanned Aircraft Systems (UAV).”.

[3] D. Glade, "Unmanned Aerial Vehicles: Implications for Military Operations," 2000.

[4] A. I. LLC., "Unmanned Aerial Target Drone Technologies for Today's Militaries," 2018. [Online]. Available: http://www.aerotargets.com/ATI_Products.htm. [Accessed: 28-Jul-2019].

[5] D. Sipos, P. Planinsic, and D. Gleich, "On drone ground penetrating radar for landmine detection," in 2017 First International Conference on Landmine: Detection, Clearance and Legislations (LDCL), 2017, pp. 1-4.

[6] J. Straub, "Unmanned Aerial Systems: Consideration of the use of Force for Law Enforcement Applications," Technol. Soc., vol. 39, pp. 100-109, Nov. 2014.

[7] A. C. Hill, "Economical Drone Mapping for Archaeology: Comparisons of Efficiency and Accuracy," J. Archaeol. Sci. Reports, vol. 24, pp. 80-91, Apr. 2019.

[8] D. I. Boeras, B. C. Collins, and R. W. Peeling, "The Use of Drones in the Delivery of Rural Healthcare," in 
Hevolutionizing Tropical Medicine, Hoboken, NJ, USA: John Wiley \& Sons, Inc., "Passenger Condition Based Route-Planning for Cognitive Vehicle System,” Int. J. Softw. Sci. Comput. Intell., vol. 10, no. 2, pp. 25-35, Apr. 2018.

[10] S. Patel, "Drone Filming: Creativity versus Regulations in Autonomous Art Systems. A Case Study.," Media- $N$, vol. 15, no. 1, pp. 17-23, Jan. 2019.

[11] T. A. Maniou and L. Papadopoulou, "Broadcast Journalism: Television," in The International Encyclopedia of Journalism Studies, Wiley, 2019, pp. 1-11.

[12] J. N. McRae, C. J. Gay, B. M. Nielsen, and A. P. Hunt, "Using an Unmanned Aircraft System (Drone) to Conduct a Complex High Altitude Search and Rescue Operation: a Case Study,” Wilderness Environ. Med., Jun. 2019.

[13] M. R. Varela et al., "Assessing climate change associated sea-level rise impacts on sea turtle nesting beaches using drones, photogrammetry and a novel GPS system," Glob. Chang. Biol., p. gcb.14526, Dec. 2018.

[14] Xiong, Xingguo, Pallis, and J. M, "Nasa Connecticut Space Grant Consortium: Balloon/Drone-based Aerial Platforms for Remote Particulate Matter Pollutant Monitoring," 2019.

[15] A. Nikulin and T. S. de Smet, "A UAV-based magnetic survey method to detect and identify orphaned oil and gas wells," Lead. Edge, vol. 38, no. 6, pp. 447-452, Jun. 2019.

[16] S. M. Shavarani, "Multi-level facility location-allocation problem for post-disaster humanitarian relief distribution," J. Humanit. Logist. Supply Chain Manag., vol. 9, no. 1, pp. 70-81, Apr. 2019.

[17] J. J. Cancela, X. P. González, M. Vilanova, and J. M. Mirás-Avalos, "Water Management Using Drones and Satellites in Agriculture," Water, vol. 11, no. 5, p. 874, Apr. 2019.

[18] M. Ammad-Uddin, A. Mansour, D. Le Jeune, E. H. M. Aggoune, and M. Ayaz, "UAV Routing Protocol for Crop Health Management," in 24th European Signal Processing Conference (EUSIPCO), 2016, pp. 1818-1822.

[19] H. Ahn, D.-T. Le, D. T. Nguyen, and H. Choo, "Real-Time Drone Formation Control for Group Display," 2019, pp. 778-785.

[20] S. AlZu'bi, B. Hawashin, M. Mujahed, Y. Jararweh, and B. B. Gupta, "An efficient employment of internet of multimedia things in smart and future agriculture," Multimed. Tools Appl., vol. 78, no. 20, pp. 29581-29605, Oct. 2019.

[21] S. P. Ahuja and N. Wheeler, "Architecture of Fog-Enabled and Cloud-Enhanced Internet of Things Applications," Int. J. Cloud Appl. Comput., vol. 10, no. 1, pp. 1-10, Jan. 2020.

[22] A. Tewari and B. B. Gupta, "Security, privacy and trust of different layers in Internet-of-Things (IoTs) framework," Futur. Gener. Comput. Syst., vol. 108, pp. 909-920, Jul. 2020.

[23] Web, "PmodGPS Reference Manual," Digilent, 2018. [Online]. Available: https://reference.digilentinc.com/_media/pmod:pmod:pmodgps-gms-u1lp_rm.pdf. [Accessed: 08-Sep-2018].

[24] H. A. Abdulqader, T. Rahman, and C. Leow, "Performance Evaluation of Localization Accuracy for a Log-Normal Shadow Fading Wireless Sensor Network under Physical Barrier Attacks,” IEEE J. Sensors, vol. 15, no. 12, pp. 30545-30570, Dec. 2015.

[25] E. Damosso, "Digital Mobile Radio Towards Future Generation Systems-Cost," 1999.

[26] N. Priyantha, A. Chakraborty, and H. Balakrishnan, "The Cricket Location-Support System," in 6th Annual International Conference on Mobile Computing and Networking - MobiCom, 2000, pp. 32-43.

[27] I. Kim, B. Kim, and J. Song, “An Efficient Location Verification Scheme for Static Wireless Sensor Networks," Sensors, vol. 17, no. 2, p. 225, Jan. 2017.

[28] R. Stoleru and J. A. Stankovic, "Probability grid: a location estimation scheme for wireless sensor networks," in 2004 First Annual IEEE Communications Society Conference on Sensor and Ad Hoc Communications and Networks, 2004. IEEE SECON 2004., 2004, pp. 430-438.

[29] N. Sundaram and P. Ramanathan, "Connectivity based location estimation scheme for wireless ad hoc networks," in Global Telecommunications Conference, 2002. GLOBECOM '02. IEEE, 2002, vol. 1, pp. 143-147.

[30] D. Pescaru and D.-I. Curiac, "Anchor Node Localization for Wireless Sensor Networks Using Video and Compass Information Fusion," Sensors, vol. 14, no. 3, pp. 4211-4224, Mar. 2014.

[31] F. Tsai, Y.-S. Chiou, and H. Chang, "A Positioning Scheme Combining Location Tracking with Vision Assisting for Wireless Sensor Networks,” J. Appl. Res. Technol., vol. 11, no. 2, pp. 292-300, Apr. 2013.

[32] F. Mekelleche and H. Haffaf, "Classification and Comparison of Range-Based Localization Techniques in Wireless Sensor Networks," J. Commun., 2017.

[33] S. P. Singh and S. C. Sharma, "Range Free Localization Techniques in Wireless Sensor Networks: A Review," Procedia Comput. Sci., vol. 57, pp. 7-16, 2015.

[34] Priya and A. Malik, "A Review on Localization Algorithms in Wireless Sensor Networks," Int. J. Comput. Sci. Eng. Technol., vol. 5, no. 6, pp. 677-682, 2014.

[35] C.-W. Fan, Y.-H. Wu, and W.-M. Chen, "RSSI-Based Localization for Wireless Sensor Networks with a Mobile Beacon," IEEE J. Sensors, pp. 1-4, Oct. 2012.

[36] S. Ravindra and S. N. Jagadeesha, "Time of Arrival Based Localization in Wireless Sensor Networks: A Linear Approach,” Int. J. Signal Image Process., vol. 4, no. 4, pp. 13-30, Aug. 2013.

[37] X. Cui, K. Yu, S. Zhang, and H. Wang, "Azimuth-Only Estimation for TDOA-based Direction Finding with ThreeDimensional Acoustic Array,” IEEE Trans. Instrum. Meas., pp. 1-1, 2019.

[38] P. Ioannides and C. A. Balanis, "Uniform Circular Arrays for Smart Antennas," IEEE Mag. Antennas Propag., vol. 47, no. 4, pp. 192-206, Aug. 2005. 
[39] M. Anwar, "Direction of Arrival in Smart Antenna using different Types of Uniform Array Configuration," Int. J. Comput. Appl., vol. 1, no. 1, pp. 2-6, 2014.

[40] Q. Wang, H.-N. Dai, Z. Zheng, M. Imran, and A. Vasilakos, “On Connectivity of Wireless Sensor Networks with Directional Antennas," Sensors, vol. 17, no. 12, p. 134, Jan. 2017.

[41] J. Liu, li Li, and W. Huazhi, "Investigation of Different Types of Array Structures for Smart Antennas," in International Conference on Microwave and Millimeter Wave Technology, 2008, pp. 1160-1163.

[42] R. Schmidt, "Multiple Emitter Location and Signal Parameter Estimation," IEEE Trans. Antennas Propag., vol. 34, no. 3, pp. 276-280, Mar. 1986.

[43] Web, “Advanced Radar Systems,” COURSE SYLLABUS (Introduction to Naval Weapons Engineering), 2019. [Online]. Available: https://fas.org/man/dod-101/navy/docs/es310/advradrsys/AdvRadr.htm. [Accessed: 02-Feb2019]. 